\title{
VARIABILIDAD ESPACIAL Y TEMPORAL DE LA COBERTURA VEGETAL DE LOS AÑOS 1984 A 2011 EN LA CUENCA HIDROGRÁFICA DEL RÍO MOXOTÓ, PERNAMBUCO, BRASIL
}

\author{
SPATIAL AND TEMPORAL VARIABILITY OF VEGETATION COVER IN THE \\ MOXOTÓ WATERSHED, PERNAMBUCO, BRASIL
}

\author{
Keyla Manuela Alencar da Silva Alves*, Maria Carolina Parodi D'avila*, \\ Ranyére Silva Nóbrega ${ }^{* *}$ y Dustyn Opazo Albarran ${ }^{* * *}$
}

\begin{abstract}
La vegetación de tipo Caatinga es predominante en la cuenca hidrográfica del Moxotó. Este tipo de vegetación ha sido deforestada de manera acelerada, debido principalmente al consumo de leña nativa, explotada para fines domésticos, industria y agricultura. El objetivo de este estudio es realizar un análisis del estado de la vegetación en el periodo de 1984 a 2011, utilizando los Índices de Vegetación por Diferencia Normalizada (NDVI) e Índice de Vegetación Ajustado al Suelo (SAVI). Ambos índices serán utilizados para identificar la vegetación de la cuenca según la densidad del área forestada. Los resultados del NDVI y SAVI fueron útiles para observar la variabilidad espacial y temporal de la vegetación en el área de la cuenca hidrográfica del río Moxotó, además fueron adaptados para generar una clasificación de la vegetación de la cuenca en categorías de densidad de área forestada. Según los resultados fue posible identificar tres tipos de densidad: baja, mediana y alta. Los resultados indicaron que la vegetación de menor densidad es la más abundante en el área de la cuenca, y que la vegetación de mayor densidad representa menos del $3 \%$ de la vegetación de la cuenca.
\end{abstract}

Palabras claves: Caatinga, NDVI, SAVI, vegetación, cuenca hidrográfica.

The Caatinga vegetation is predominant in the Moxoto basin hidrology. This type of vegetation has been cleared at an accelerated rate, mainly due to the consumption of native wood, exploited for domestic industrial and agricultural purposes. The aim of this study is to analyze the state of the vegetation in the period 1984-2011 using the Vegetation Index Normalized Difference-NDVI and Soil-Adjusted Vegetation Index - SAVI. Both indexes will be used to identify the vegetation of the basin according to its density of forested area. The NDVI and SAVI results were adapted to generate a watershed vegetation classification out of vegetal density categories. According to the results, it was possible to identify three types of vegetation density of forested area: low, medium and high. The results indicate a profuse presence of the lower density vegetation in the watershed and that the higher density vegetation is less than $3 \%$ of the vegetation in the watershed.

Key words: Caatinga, NDVI, SAVI, vegetation, basin hidrology.

\section{Introducción}

Los cambios del uso de la tierra ocurren con más rapidez en los trópicos que en otras regiones del planeta (Hansen et al., 2013). En el semiárido brasileño, ubicado en la región tropical, una de las principales actividades antrópicas que contribuyen a la deforestación de la vegetación de tipo Caatinga es la agricultura. En toda la región Nordeste esta actividad está con fuerza sostenida en la explotación de los recursos naturales, y principalmente en lo que se refiere a la extracción excesiva de la cobertura vegetal. Desde el período colonial la cobertura vegetal es explotada. La retirada de la vegetación para el plantío, y también utilización de la vegetación como insumos energéticos para la industria (Paes Silva, 2000).

La vegetación de Caatinga es predominante en la cuenca hidrográfica del río Moxotó, así como en casi todo el semiárido brasileño (Giulietti et al.,

\footnotetext{
* Universidad Tecnológica Metropolitana. Departamento de Industria. Santiago, Chile. Correos electrónicos: keyla.dasilva@ utem.cl; cparodi@utem.cl

** Universidade Federal de Pernambuco. Departamento de Ciencias Geográficas. Cidade Universitária, Recife, Brasil. Correo electrónico: ranyere.nobrega@ufpe.br

*** Universidad de Chile. Departamento de Geografía, Laboratorio de Territorio y Medio Ambiente, Santiago, Chile. Correo electrónico: dustyn.opazo@gmail.com
} 
2003). Este tipo de vegetación está caracterizada por una capa arbórea de 10 a $15 \mathrm{~m}$ de altura, con una cobertura bastante densa, entretanto no es completamente cerrada, por lo general; y una capa arbustiva de 4 a $6 \mathrm{~m}$ de altura. La vegetación de Caatinga abarca un gran número de formaciones y asociaciones vegetales, fisionómica y florísticamente diferentes y presenta un aspecto verde después de los primeros días de lluvia (Alves et al., 2013), entretanto cuando hay pérdida de la cobertura vegetal se domora una media de 10 a 15 años para recuperar más de $90 \%$ de la biomasa original (Andrade y Oliveira, 2004).

La cuenca hidrográfica del río Moxotó está ubicada en una región semiárida, caracterizada por la expresiva variabilidad estacional (inter e intraanuales) de la precipitación, intensa extracción de la cobertura vegetal, y con altos niveles de fragilidad ambiental (Assis et al., 2012; Belfort y Barbosa, 2011). La cuenca del Moxotó también pasó por intensos procesos de intervenciones antrópicas que provocaron impactos y potencializaron la vulnerabilidad. La pérdida de vegetación en la cuenca está vinculada a dos posibles causales que son la intensa variabilidad pluvial y la retirada de vegetación para consumo energético (Alves y Nobrega, 2017).

La dependencia de la población y demás sectores de la economía con la biomasa como fuente de energía representa alrededor de $50 \%$ del consumo de energía primaria en el semiárido (Ministério do Meio Ambiente, 2008). Todavía las especies forestales de Caatinga son usadas en la producción de leña y carbón, y esa producción ha sido intensiva y desordenada (Riegelhaupt y Pareyn, 2010). En la región semiárida del Nordeste brasileño la leña aun representa una fuente energética importante para la pequeña y mediana industria. Entre las principales industrias consumidoras de leñas se destacan las mineras de yeso y las fábricas de ladrillo. Estas industrias, junto con la agricultura y la actividad pecuaria, forman la base de la economía en el semiárido. La leña utilizada por la industria en el semiárido proviene de la extracción intensiva en zonas de vegetación nativa, la retirada de la madera en estas zonas es ilegal y sin ninguna sustentabilidad, causando desforestación e impacto en la biodiversidad (Reis et al., 2017; Cardoso et al., 2015; Pernambuco, 2007; Gomes, 2006).
Eliminar la cobertura vegetal genera severos impactos en el suelo. El terreno expuesto posee mayor capacidad de absorción de la radiación solar, de esta manera aumenta la temperatura local y acelera la evapotranspiración. Los impactos de las precipitaciones sobre superficies expuestas disminuyen la capacidad de almacenamiento de agua. (Guimarães et al., 2016).

Ahlstrom et al. (2015) clasifican las zonas semiáridas como "sumidero de carbono", por tratarse de ecosistemas terrestres capaces de absorber grandes cantidades de $\mathrm{CO}_{2}$. Aún según los autores, en un análisis de la dinámica de la vegetación, se señala que los ecosistemas semiáridos pueden absorber más $\mathrm{CO}_{2}$ que las forestas tropicales.

Entre las principales técnicas de teledetección aplicadas al procesamiento de imágenes satelitales para el análisis de la dinámica vegetal se destacan el Índice de Vegetación por Diferencia Normalizada o NDVI (sigla en inglés de Normalized Difference Vegetation Index) (Georganos et al., 2017; Tian et al., 2015; Zhao et al., 2015; Fonseca, 2000; Myneni et al., 1995) y el Índice de Vegetación Ajustado al Suelo o SAVI (sigla en inglés Soil Adjusted Vegetation Index) (Bezerra et al., 2017; Oliveira et al., 2009; Heute, 1988).

El NDVI funciona como estimador de radiación utilizada dentro del proceso de fotosíntesis que ocurre en las hojas, y monitoriza la variabilidad periódica de las plantas (Duan, et al., 2017a; Stockli \& Vidale, 2004; Stockli, 2005). Este índice presenta excelente respuesta en el análisis de los cambios en la cantidad de la biomasa verde, contenido de clorofila y estrés hídrico (Pizon et al., 2014; Liang, 2004). El SAVI fue desarrollado por Huete (1988) con el propósito de suavizar la influencia del suelo, aplicando un factor de ajuste L que actúa en la corrección de las intervenciones de suelo y de las condicionas atmosféricas en el cálculo del índice de vegetación.

El objetivo de este estudio es analizar la variabilidad de la cobertura vegetal por medio del diagnóstico de pérdida y ganancias de vegetación arbórea y arbustiva durante el período de 1984 a 2011, mediante la aplicación de los índices de vegetación NDVI y SAVI. 


\section{Metodología}

\section{Área de estudio}

La cuenca hidrográfica del río Moxotó está totalmente inserta en los límites del semiárido del Nordeste brasileño. Se sitúa en la porción central del estado de Pernambuco y en la parte oeste del estado de Alagoas, alcanzando un área de aproximadamente $9.752,71 \mathrm{~km}^{2}$ (siendo $8.778,05 \mathrm{~km}^{2}$ en Pernambuco y $974,66 \mathrm{~km}^{2}$ en Alagoas).

El clima de la cuenca del Moxotó, según la clasificación climática de Köppen, es BShW', que corresponde al tipo cálido y seco, con total pluviométrico anual medio de $435 \mathrm{~mm}$ y lluvias concentradas de enero a mayo. La temperatura, la humedad relativa y la evapotranspiración potencial media anual son de $25^{\circ} \mathrm{C}, 55 \%$ y $3.008 \mathrm{~mm}$, respectivamente (Figura 1). (Alves y Nobrega, 2016; Alves y Nobrega, 2017; Perrazzo, 2002).

Este tipo de clima se configura por su extrema variabilidad e influencias de eventos climáticos extremos; por ejemplo, El Niño/La Niña. La región semiárida presenta una taza de precipitación relativamente alta, pero la distribución estacional de las lluvias está concentrada en pocos meses del año (período lluvioso), y todos los demás meses son de sequía. Otro aspecto de la climatología de la región que contribuye con la escasez del agua son los altos niveles de evapotranspiración que provocan déficit en el acúmulo de agua (Assis et al., 2014; Angelotti et al., 2009).

La geología de la cuenca del Moxotó es compleja, las nacientes están sobre estructuras cristalinas asociadas a la ladera occidental de la meseta de la Borborema. En el inicio del curso medio del río principal surgen nacientes sobre morfología tabuliformis y homoclinales asociados a la meseta de la cuenca del Jatobá (Oliveira, 2009). La cuenca posee una altitud que varía entre 200 y $1.100 \mathrm{~m}$ (Figura 2).

\section{Descarga, registro y corrección geográfica de las imágenes}

Para el análisis propuesto fueron utilizadas 84 imágenes del satélite Landsat 5 (TM), de la órbita y punto 215/66, compuestas por siete bandas espectrales, adquiridas gratuitamente mediante el catálogo de imágenes del Instituto de Pesquisas Espaciais - Brasil (INPE).
Todas las imágenes seleccionadas son de octubre, noviembre y diciembre, meses del período seco. Fueron preferidas imágenes de este período por poseer la menor cantidad de nubes, para tener mejor visualización de la vegetación. La presencia de nubes dificulta bastante la identificación de objetos en la superficie, además de influir en el resultado del índice (Tucker et al., 1991). Por lo mismo, y teniendo en cuenta que en este período la vegetación de la zona presenta, naturalmente, muy baja densidad, la preferencia fue por el período citado para que se pudiera observar con exactitud la ejecución de la técnica sin influencia de nubes. Además, se eligió las imágenes del período de 1984 a 2011 porque esta es la única serie de imágenes Landsat continuas.

\section{Calibración radiométrica}

Esta etapa se constituye en el cálculo de la radiancia espectral de cada banda, o sea, la conversión radiométrica donde el número digital de cada pixel de la imagen es convertido en la radiación espectral monocromática (Silva, 2009). Según Markahm y Baker (1987), este cálculo representa la radiación solar reflejada por cada pixel, por unidad de área, de tiempo, de ángulo sólido y longitud de onda, medida al nivel del satélite LANDSAT5 TM, para las bandas 1 a 7 (Ecuación 1).

$$
\mathrm{L}_{\lambda \mathrm{i}}=\mathrm{a}_{\mathrm{i}}+\frac{\mathrm{b}_{\mathrm{i}}-\mathrm{a}_{\mathrm{i}}}{255}=\mathrm{ND} \quad \text { Ecuación } 1
$$

Donde a y b son las radiaciones espectrales mínima y máxima, respectivamente, ND es la intensidad del pixel, e i corresponde a las bandas de imagen. La radiancia calculada es la conversión radiométrica del número digital en valor monocromático, en este caso el valor de la radiancia que es presentada por cada pixel corresponde a la medida de las radiaciones de todos los cuerpos identificados en el área de la imagen.

\section{Cálculo de reflectancia}

Es definido como la razón entre el flujo de la radiancia solar reflejada y el flujo de la radiancia solar incidente (Ecuación 2):

$$
\rho_{\lambda \mathrm{i}=\frac{\pi \cdot \mathrm{L}_{\lambda \mathrm{i}}}{\mathrm{k}_{\lambda \mathrm{i}} \cdot \cos \mathrm{Z} \cdot \mathrm{d}_{\mathrm{r}}}}
$$

Ecuación 2 


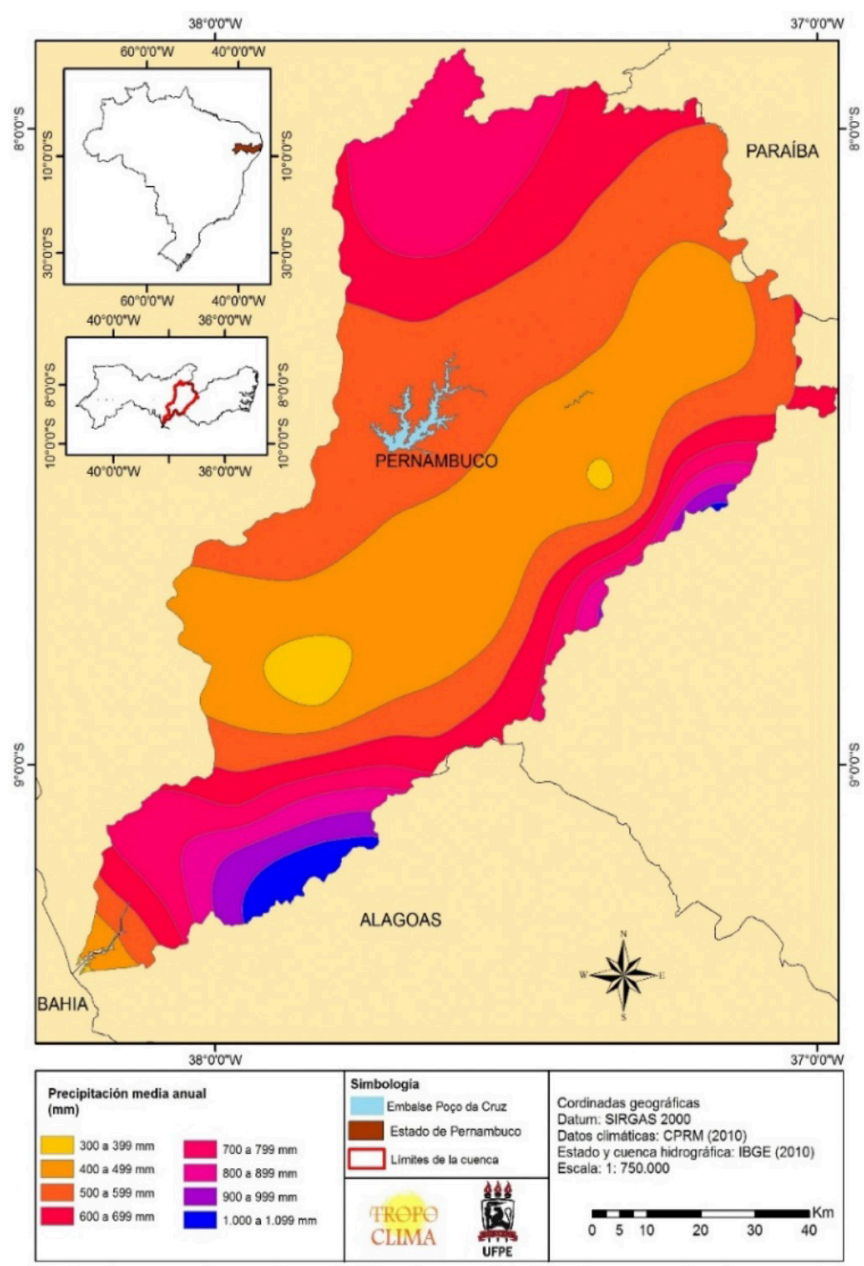

Figura 1. Mapa de localización y precipitaciones de la cuenca.

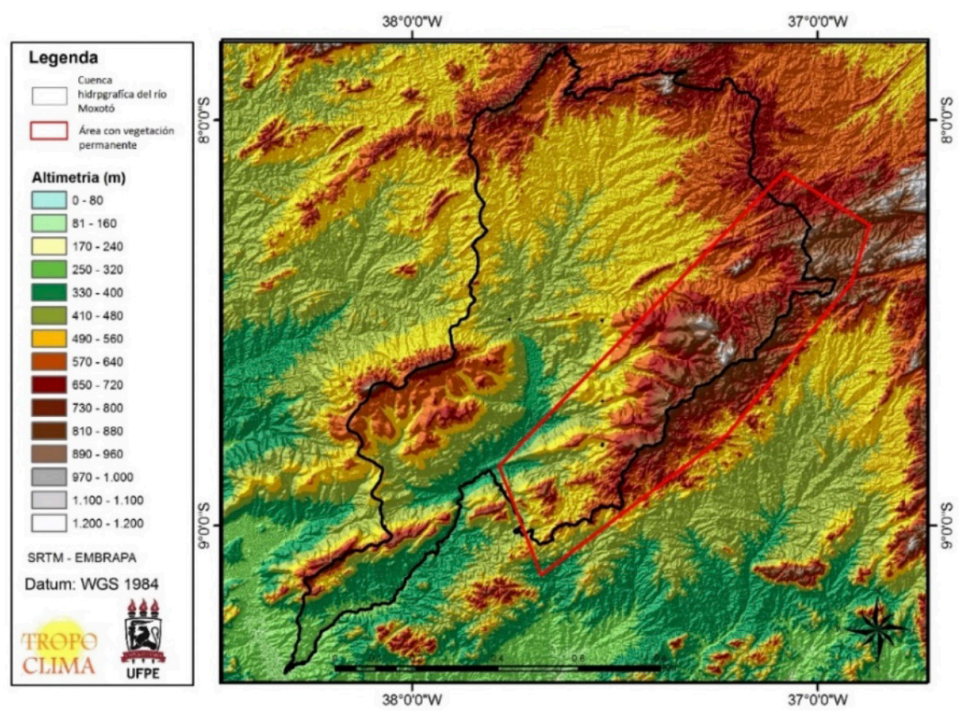

Figura 2. Mapa altimétrico de la cuenca hidrográfica del río Moxotó. 
Donde $\mathrm{L}_{\lambda_{\mathrm{i}}}$ es la radiación espectral de cada banda, $\mathrm{k}_{\lambda \mathrm{i}}$ es la irradiación solar espectral de cada banda en el topo de la atmósfera, $\mathrm{Z}$ es el ángulo cenital y $\mathrm{d}_{\mathrm{r}}$ es el cuadrado de la razón entre la distancia tierrasol $\left(r_{o}\right)$ y tierra-sol $(r)$, en un determinado día del año. La reflectancia espectral de una determinada superficie es extremadamente compleja, una vez que la superficie de la Tierra, en general, es heterogénea y agrega diversos elementos (Fiorio et al., 2001).

\section{Cálculo del Índice de Vegetación por Diferencia Normalizada (NDVI)}

El índice NDVI fue obtenido por la razón de las diferencias entre la reflectancia del infrarrojo cercano y el rojo (Ecuación 3).

$$
\mathrm{NDVI}=(\mathrm{A}-\mathrm{B}) /(\mathrm{A}+\mathrm{B}) \quad \text { Ecuación } 3
$$

Donde A es la reflectancia en el canal infrarrojo próximo; B es la reflectancia en el canal rojo. Por tanto, al analizar la curva de la reflectancia de la vegetación el desvío observado entre el canal rojo y el infrarrojo próximo constituye una variable sensible a la presencia de la vegetación verde (Duan et al., 2017). La respuesta espectral de la vegetación en el canal infrarrojo está directamente relacionada a la concentración de la clorofila, mientras la respuesta espectral del canal infrarrojo es controlada por el índice de área foliar y la densidad de vegetación verde (Crusiol et al., 2016; Foster et al., 2016; Major et al., 1990). La combinación de esos dos dominios espectrales permite diferenciar los distintos tipos de vegetación, determinando fotosintéticamente la biomasa activa por medio de la densidad de la cobertura vegetal, y diferenciando la vegetación de los demás elementos presentes en la superficie (Duan et al., 2017b; Oliveira y Galvanício, 2009; Tucker, 1978; Rouse et al., 1974).

\section{Cálculo del Índice de Vegetación Ajustado al Suelo-SAVI}

Este índice surge a partir de un reajuste realizado por Heute (1988) a la fórmula original del NDVI, incorporando a la fórmula original el factor de efecto del suelo, manteniendo el valor del NDVI entre $-1 \mathrm{y}+1$. Este índice está expresado por la siguiente ecuación 4 :

$$
S A V I=\frac{\mathrm{Nir}-\mathrm{Red}}{\mathrm{Nir}+\operatorname{Red}+\mathrm{L}}(1+L) \quad \text { Ecuación } 4
$$

Nir y Red corresponde respectivamente a las bandas infrarroja cercana y roja, L es la constante, cuyo valor aplicado para el área de estudio según literatura es de 0,5 (Silva et al., 2005; Accioly et al., 2002; Boegh et al., 2002).

\section{Clasificación supervisada}

Para clasificar el efecto los resultados de los índices se tomaron como base la clasificación supervisada propuesta por Lourenço y Landim (2004) para el NDVI y para el SAVI la clasificación propuesta por Oliveira et al. (2009). Adaptado al estudio por los autores, los valores positivos más altos del NDVI y del SAVI fueron asociados a la mayor densidad de cobertura vegetal, y los valores positivos más bajos fueron asociados a la menor densidad de cobertura vegetal, superficies expuestas, cuerpos de agua (Tabla 1).

Según el informe de Biodiversidad de la CaatingaMinisterio del Medio Ambiente de Brasil (2003), la región central del semiárido en Pernambuco (donde está ubicada la cuenca del Moxotó) presenta predominantemente el dominio de la vegetación xerófila, también presentando áreas con dominio de vegetación xerófila y pequeñas islas húmedas (Brejos) ${ }^{1}$.

Tabla 1: Valores do Índices x Clases.

\begin{tabular}{cccc}
\hline Valores NDVI & Clases & Valores SAVI & Clases \\
\hline-1 a $-0,32$ & Agua & $-0,243-0$ & Agua \\
$-0,31$ a $-0,059$ & Áreas húmedas & $0,001-0,226$ & Suelo expuesto \\
$-0,058$ a 0,0039 & Suelo expuesto & $0,227-0,341$ & Vegetación poco densa \\
0,004 a 0,12 & Vegetación poco densa & $0,342-0,405$ & Vegetación medio densa \\
0,13 a 1 & Vegetación densa & $0,406-0,688$ & Vegetación muy densa \\
\hline
\end{tabular}


Considerando que el objetivo es analizar apenas la vegetación arbórea y arbustiva, la clasificación supervisada fue adaptada de acuerdo con el perfil vegetal de la Caatinga según la fisionomía (Figura 3).

\section{Resultados}

Según los resultados del NDVI es posible notar que la topografía ejerce una gran influencia en la distribución espacial de la cobertura vegetal. La vegetación nativa se presenta más conservada en las áreas altitudinales más elevadas (Este de la cuenca), en las áreas de cerros islas y relieves residuales de la cuenca sedimentar de Jatobá. Analizando este resultado y comparando con la caracterización de tendencias pluviométricas de la cuenca, realizada por Alves y Nobrega (2017), aplicando el método Mann-Kendall, se observa que el sector Este es también el área donde presenta tendencia significativamente creciente para el mismo periodo analizado en este estudio $(Z=2,20$-precipitación media $=92,9 \mathrm{~mm}$ ). Los demás sectores de la cuenca no presentan tendencias de precipitaciones positivas significativas. Por tanto, el sector donde se observa mayor densidad de cobertura vegetal coincide con la zona más elevada de la cuenca y la que posee las mayores tazas de precipitaciones concentradas entre marzo y julio. Este análisis indica que la variabilidad pluviométrica representa un factor condicionante para la distribución y conservación de la vegetación de la cuenca.

Los resultados de los índices NDVI y SAVI fueron semejantes en relación con su distribución espacial, lo que es observado por medio de las imágenes resultantes de los cálculos realizados. No obstante, al obtener el valor de área de cada uno de los tres rangos de densidad de cobertura vegetal analizado, fue posible apreciar que los resultados de SAVI entregan mayores detalles en cuanto a la diferencia de densidad, ya que el NDVI imposibilita la mejor percepción de la cantidad de vegetación por rango. La vegetación de mediana y alta densidad fue mejor identificada por el SAVI cuando se comparan los resultados con el NDVI de la cuenca (Figura 4).

Según los resultados, se definieron tres categorías de densidad de la vegetación arbórea y arbustiva:

- Categoría 1: NDVI $(0,25-0,50)$ y SAVI $(0,401$ 0,667): vegetación de baja densidad;

- Categoría 2: NDVI $(0,50-0,75)$ y SAVI $(0,668$ $0,933)$ : vegetación de mediana densidad;

- Categoría 3: NDVI (0,75-1) y SAVI (0, 933-1): vegetación de alta densidad.

Fueron calculados el área de cada categoría para cada año de la serie en análisis, luego se obtuvo el porcentaje del área y a partir de estos valores, extraídos de las imágenes resultantes, fue posible calcular el crecimiento y la pérdida de vegetación de cada categoría.

\section{Categoría 1: baja densidad NDVI $(0,25-0,50)$ y SAVI (0,401-0,667)}

Esta categoría está compuesta por vegetación arbórea-arbustiva de baja densidad, y según los resultados es el tipo de vegetación más abundante en la cuenca. Los resultados del gráfico abajo representan cuantitativamente (porcentaje de área) la presencia de esta categoría vegetal en el perímetro de la cuenca. En el análisis comparativo entre los resultados de ambos índices para esta categoría se observa semejanza entre los valores. Tanto el NDVI como el SAVI apuntan que la categoría de baja densidad de cobertura vegetal es la que más se mantiene conservada durante los 27 años de análisis. Con excepción del 2002 que presenta discrepancia entre los resultados, siendo el único año atípico de toda la serie temporal.
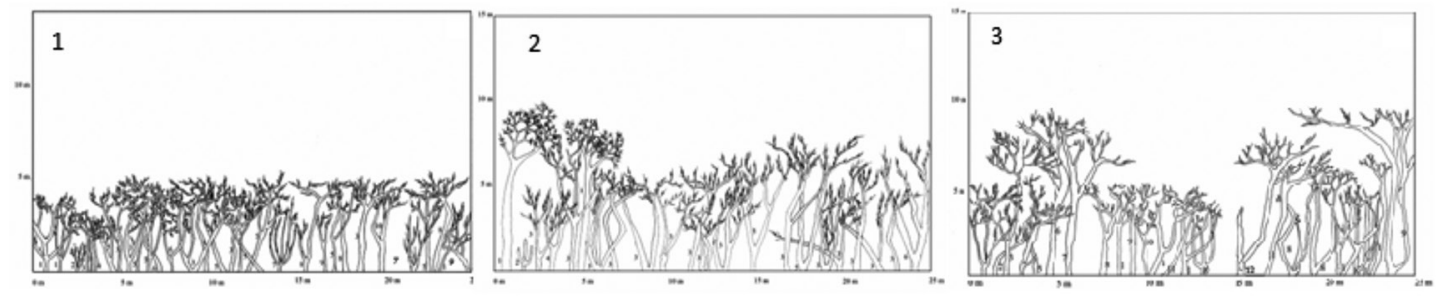

Figura 3. Caatinga arbórea-arbustiva de baja densidad (1), mediana densidad (2) y alta densidad (3). 

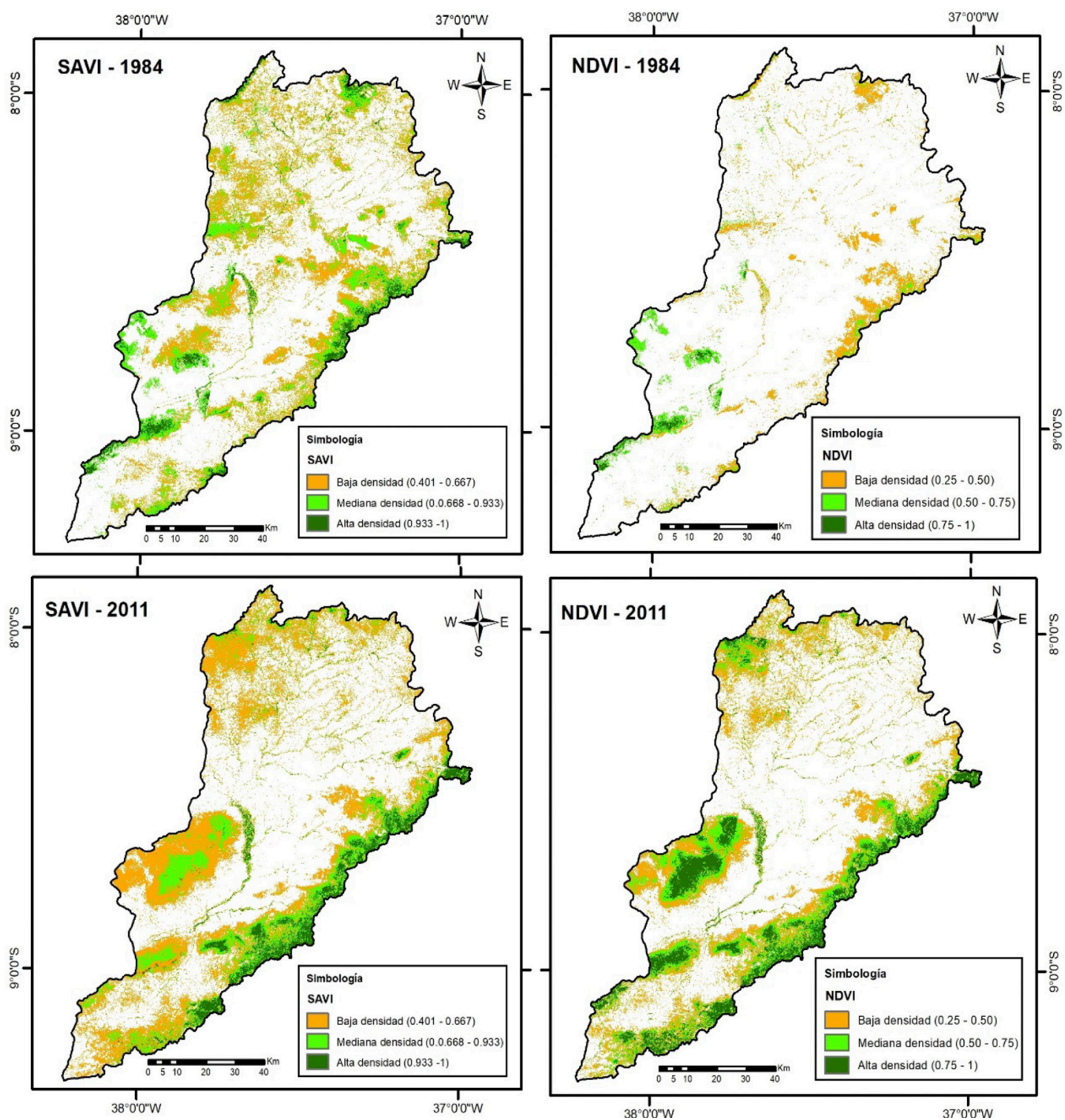

Figura 4. Resultados del NDVI y SAVI de la serie de 1984 a 2011.

El SAVI identificó en este año apenas 35\% del área vegetada como poco densa, y el NDVI 99\% (Figura 5). Buscando identificar los factores que posiblemente influenciaron en los resultados del 2002, se plantea que: según la literatura (Nobrega y Santiago, 2014), 2002 fue un año de El Niño riguroso, que para esta zona significa extrema sequía, lo que provoca una disminución de la vegetación, principalmente de la vegetación poco densa por ser menos resistente a los largos periodos de escasez de precipitaciones y sequía severa. Además, el
SAVI identificó con mayor detalle la densidad de cobertura de la vegetación del 2002. Observando los resultados de este año para ambos índices, se identifica que es posible que el resultado del NDVI no diferencie con claridad la baja densidad de la mediana densidad vegetal. La tasa de variación (crecimiento o pérdida de vegetación) de esta categoría es positiva para ambos índices. Para la serie en análisis el SAVI presentó $0,525 \%$ y el NDVI 0,521\% de crecimiento de la vegetación poco densa (Figura 6). 


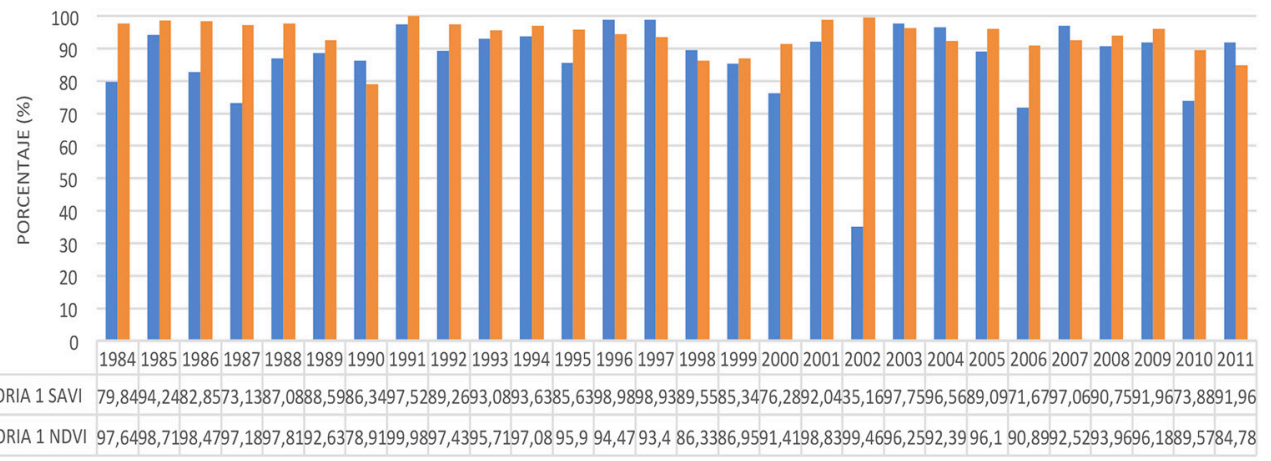

Figura 5. Valores cuantitativos de la cobertura vegetal de la cuenca.

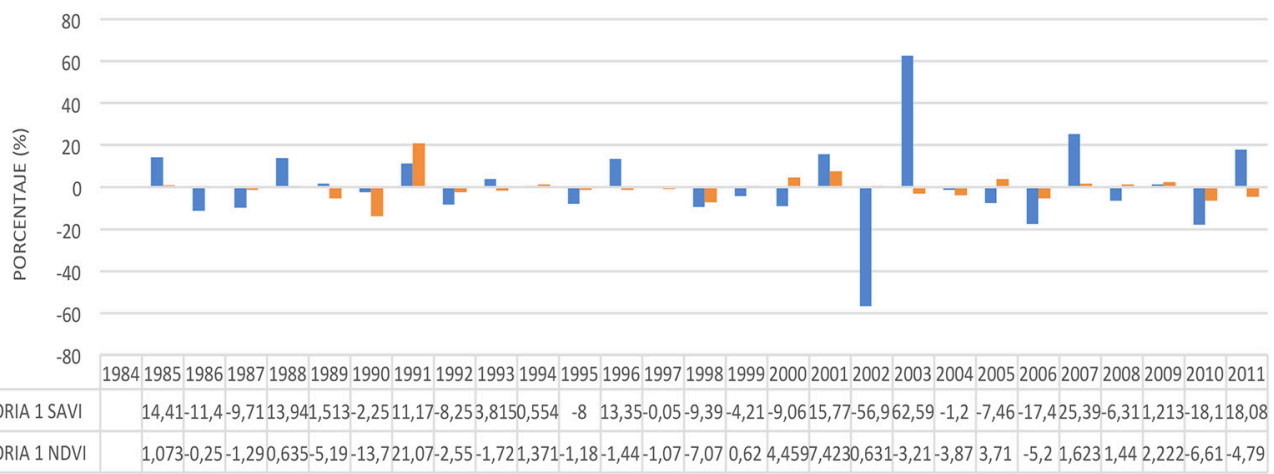

Figura 6. Valores cuantitativos de la variabilidad anual de la cobertura vegetal.

\section{Categoría 2: mediana densidad NDVI (0,50- 0,75) y SAVI (0,668-0,933)}

La categoría 2 representa la vegetación arbóreaarbustiva de densidad mediana, siendo también la segunda más abundante en el perímetro de la cuenca. Se constata por medio de los análisis comparativos de los resultados de ambos índices para esta categoría que el SAVI distinguió con más detalles la vegetación de mediana densidad de cobertura de las demás categorías. Según las imágenes con los resultados de los índices fue posible observar que los efectos del NDVI identificaron como vegetación poco densa lo que en las imágenes del SAVI se presentaba como vegetación de mediana densidad. Los porcentajes de área ocupada por esta categoría variaron de $62 \%$ a $1 \%$ en los resultados del SAVI, y de $21 \%$ a $0,249 \%$ en los resultados del NDVI (Figura 7). Entre los años analizados en la serie se pudo observar que en el 2002 la vegetación de mediana densidad se mantuvo más conservada que las demás, presentando valores positivos, siendo el único año atípico de toda la serie, por presentar porcentajes de área ocupada superiores a los 50\%. La tasa de variación de esta categoría también fue positiva, pero no presentó resultados semejantes entre los índices, como en la categoría anterior. El SAVI presentó una tasa de crecimiento positivo de la vegetación de $3,2 \%$, mientras que el NDVI presentó una tasa de $7,1 \%$. Esta fue la categoría que presentó los mejores resultados de la tasa de variación, pudiendo concluir que para esta serie temporal en análisis es la vegetación de mediana densidad la que presentase mejor conservación en los años analizados (Figura 8).

\section{Categoría 3: alta densidad NDVI (0,75-1) y SAVI (0, 933-1)}

Esta es la categoría que presenta la vegetación arbórea-arbustiva más densa, y que a su vez es la que presenta los valores porcentuales más bajos. 


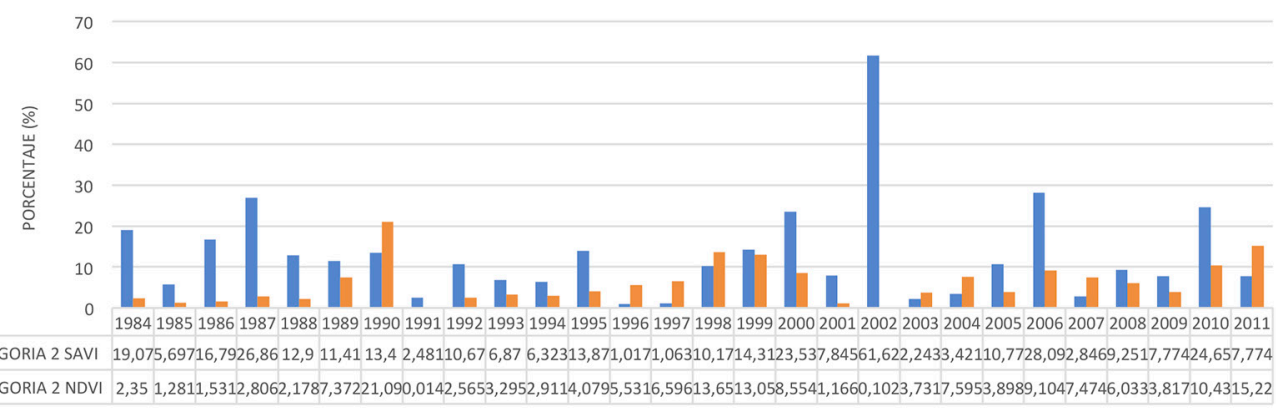

Figura 7. Valores cuantitativos de la cobertura vegetal de la cuenca.

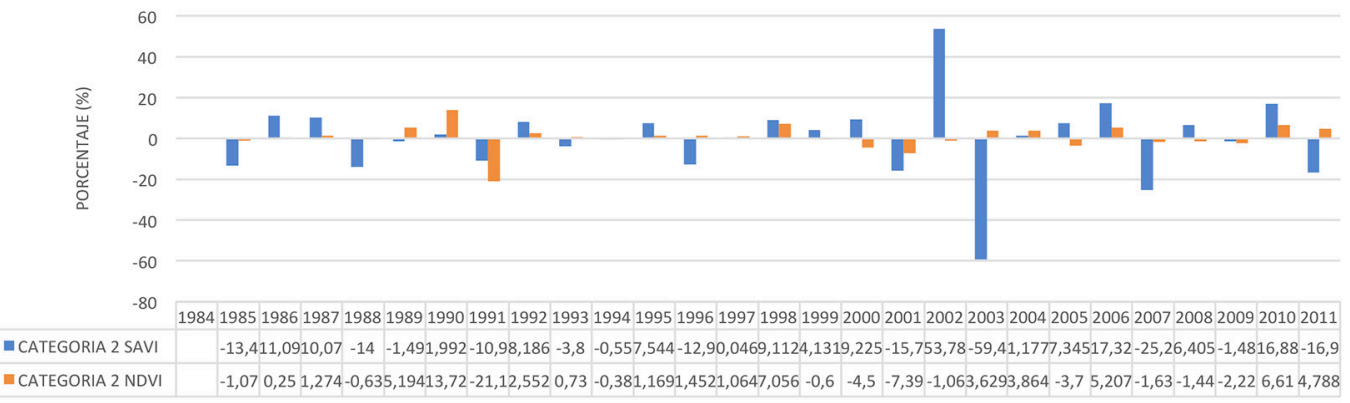

Figura 8. Valores cuantitativos de la variabilidad anual de la cobertura vegetal.

Según los resultados, la cuenca no posee más que $1 \%$ de alta densidad de cobertura en índice NDVI, y $3 \%$ en el SAVI. La cuenca posee una muy baja presencia de vegetación densa, presentando pérdidas en varios años (no consecutivos) de la serie temporal analizada. La vegetación densa se ve concentra en el Este de la cuenca, en una zona más elevada. Es posible observar que en todos los años la mayor área ocupada por esta categoría vegetal se encuentra en este sector de la cuenca. Los resultados de los índices NDVI y SAVI son bastante semejantes en cuanto al porcentaje de área ocupada por esta categoría de vegetación (Figura 9). La tasa de variación de crecimiento o pérdida también presentaron resultados semejantes, ambos índices indican pérdida de vegetación en esta categoría. El SAVI presentó una tasa negativa de $-5,13 \%$ indicando pérdida de la vegetación en el periodo analizado, y el NDVI presentó una taza de $-4,91 \%$ (Figura 10).

\section{Conclusiones}

Según la metodología propuesta por este estudio, y los respectivos resultados y análisis comparativos entre los índices NDVI y SAVI, se concluye que la vegetación nativa de la cuenca hidrográfica de Moxotó durante el periodo de 1984 a 2011 se encontró más conservada en las áreas más elevadas de la cuenca, donde la vegetación más abundante estuvo más preservada en las áreas de mayor altitud. También se puedo concluir que la vegetación menos densa es la más cuantiosa en la cuenca, y que en los años de El Niño severo (2002) la categoría de vegetación mediana fue la que se mantuvo más conservada en comparación con las demás categorías. La vegetación de Caatinga se caracteriza por ser resistente a los largos periodos de sequía, entretanto, son las de mayor densidad que mejor enfrenta los periodos de sequía extrema, como los años de El Niño severos.

También que el SAVI presentó mejores resultados en relación con la diferenciación de la categoría de vegetación de mediana densidad, siendo esta categoría la que presentó los mejores resultados referente al porcentaje de área ocupada y la tasa de variación de la vegetación, indicando en ambos índices crecimiento de la vegetación de mediana densidad. Por tanto, este estudio define que la aplicación del SAVI para la identificación de 


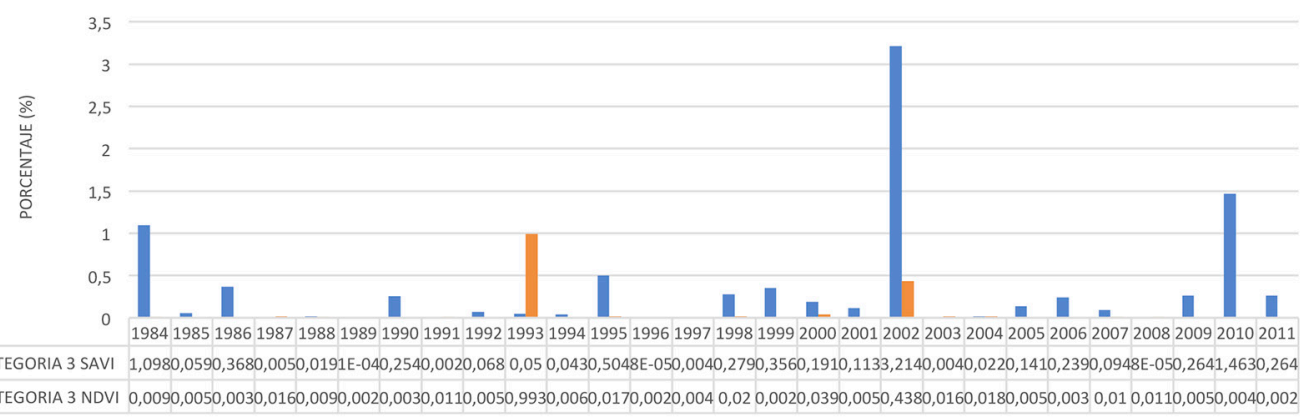

Figura 9. Valores cuantitativos de la cobertura vegetal.

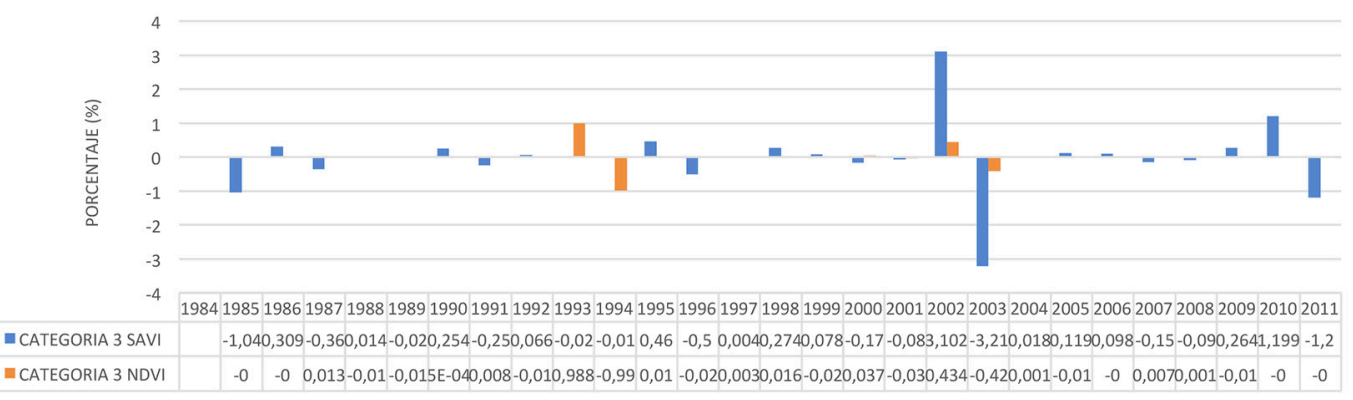

Figura 10. Valores cuantitativos de la variabilidad anual de la cobertura vegetal.

la dinámica vegetacional de tipo Caatinga arbóreaarbustiva cumplió mejor la función para alcanzar el objetivo del trabajo.

La vegetación poco densa es de menor interés comercial en la zona, ya que esta posee menor densidad de masa vegetal, pero está expuesta a la deforestación para implementación y desarrollo de las actividades agrícolas y agropecuarias. A pesar de los factores antrópicos que contribuyen con la retirada de la vegetación menos densa, esta se pudo mantener más conservada en la serie temporal en análisis.

Según los resultados, se constató que la vegetación de menor densidad de cobertura es la más abundante en el área de la cuenca. Ambos índices indicaron valores de crecimiento positivos para esta categoría, pero poco expresivos: NDVI $=0,521 \%$ y $\mathrm{SAVI}=0,525 \%$. La vegetación de densidad mediana es la segunda más abundante. Los resultados de la tasa de variación del NDVI $=7,1 \%$ indican que hubo un crecimiento positivo durante el periodo analizado, mientras que el SAVI $=3,2 \%$ presentó un valor inferior, pero igualmente positivo, apuntando crecimiento de la vegetación de mediana densidad. La vegetación de mayor densidad y la menos abundante, los resultados de ambos índices indican pérdida de la vegetación de alta densidad durante el periodo analizado, el NDVI $=-4,91 \%$ y SAVI $=-5,13 \%$.

La variabilidad pluviométrica ejerce función condicionante natural en la distribución y conservación de la vegetación nativa. No obstante, es la extracción intensa y no regulada de la vegetación para consumo energético, agricultura y pecuaria la que altera la dinámica natural de la vegetación, y que deben ser controladas por los órganos responsables. Considerando la alta fragilidad de los suelos en la zona semiárida, y la importancia de la vegetación en el control de la erosión y salinización de los suelos de tal zona, este estudio recomienda el uso de geotecnologías y teledección como medio de monitoreo de la vegetación de Caatinga, como alternativa rápida y menos costosa para uso en las municipalidades correspondientes del área de estudio. De esta manera se pueden acotar los tiempos de espera de datos geoprocesados, ya que estos están a cargo de órganos federales y tardan en transpasarlos hasta los municipios. 


\section{Agradecimiento}

A la Fundação de Amparo a Ciência e Tecnologia del estado de Pernambuco (Brasil) por financiar esta investigación. Al Laboratorio de Climatología Tropical y Eventos Extremos y a la Universidad Tecnológica Metropolitana (Chile) por el apoyo a esta investigación.

\section{Referencias Citadas}

Ahlström, A.

2015 "The dominant role of semi-arid ecosystems inthe trend and variability of land CO2 sink". Science, 1-19.

Alves, K. A., \& Nobrega , R. S.

2017 "Tendencias pluviométricas y concentración estacional de precipitaciones en la cuneca hidrográria del río Moxotó, Pernambuco, Brasil". Revista Geográfica de América Central, 295-313.

Alves, F. T., Ferreira, R. L., Silva, J. A., Marangon, L. C., \& Céspedes, G. H.

2013 "Regeneração natural de uma área de caatinga no Sertão pernambucano, Nordeste do Brasil". CERNE, 229-235.

Andrade, J. B., \& Oliveira, T. S.

2004 "Análise espaco-temporal do uso da terra em parte do semiárido cearense". Revista brasileira de ciencia do solo, 393-401.

Angelotti, F., Sá, I. B., Menezes, E. A. \& Pellegrino, G. Q. 2009 Mudanças climáticas e desertificação no semi-árido brasileiro. Empresa Brasileira de Pesquisa Agropecuária, Petrolina.

Assis, J. M. O., Calado, L. O., Souza, W. M. \& Sobral, M. C. 2014 "Mapeamento do uso e ocupação do solo no município de Belém de São Francisco - PE nos anos de 1985 e 2010". Revista Brasileira de Geografia Física, 7(5), 859-870.

Belfort, C. F. \& Barbosa, I. R.

2011 Avaliação da Fragilidade Ambiental da Bacia Hidrográfica do Rio Moxotó em Pernambuco Utilizando Geoprocessamento. Simpósio brasileiro de sensoriamento remoto (p. 1137). Curitiba: INPE.

Crusiol, L. G., Carvalho, J. F., Sibaldelli, R. N., Neiverth, W.,

Rio, A., Ferreira, L. C., Farias, J. R.

2016 "NDVI variation according to the time of measurement, sampling size, positioningof sensor and waterregimein different soybean cultivars". Precis. Agric., 1-21.

Cardoso, J. J., Barros Junior, A. P., Reis Neto, A. F., Alves, G.

S., Souza, W. M., \& Araujo, M. B.

2015 Uso Energético da Lenha nas Casas de Farinha no Municipio de Lajedo no estado de Pernambuco. XII Congresso Nacional de Meio Ambiente (págs. 1-15). Pocos de Caldas: CNMA.

Da Silva, B. B., Lopes, G. M., \& De Azevedo, P. V.

2005 "Balanço de radiação em áreas irrigadas utilizando imagens Landsat 5-TM". Revista Brasileira de Meteorologia, 20(2), 243-252.

Da Silva, C. A. V., da Silva, H. A., de Oliveira, T. H., \&

Galvincio, J. D.

2009 Uso do Sensoriamento Remoto através de Índices de Vegetação NDVI, SAVI e IAF na microrregião de ItamaracáPE. Anais XIV Simpósio Brasileiro de Sensoriamento Remoto. Natal, Brasil.

Duan, T., Chapman, S. C., Guo, Y., \& Zheng, B.

2017a "Dynamic monitoring of NDVI in wheat agronomy and breeding trials using an unmanned aerial vehicle". Field Crops Research, 71-80.
Duan, T., Zheng, B., Guo, W., Nimomiya, S., Guo, Y., \& Chapman. 2017b "Comparison of ground cover estimates from experiment plots in cotton, sorghum and sugarcane based on images and orto-mosaic capture by UAV'. Funct. Plant Biol., 169-184.

Gomes, L.

2006 Procedencia e consumo de lenha das casas de farinha nos limites do Parque Nacional Serra de Itabaiana - Sergipe. II Seminario de área protegidas e inclusao social (págs. 1-4, Rio de Janeiro: UFRJ.

Huete, A. R.

1988 "A soil-adjusted vegetation index (SAVI)". Remote Sensing of Environment, 25(3), 295-309.

Fiorio, P. R., Dematte, J. A., \& Nanni, M. R.

2001 Dados radiométricos nos níveis de laboratório, campo e orbital na discriminacao de solos desenvolvidos numa topossequencia. X Simpósio Brasileiro de Sensoriamento Remoto (págs. 1393-1397). Paraná: Anais SBSR.

Fonseca, E. L.

2000 Caracterizacao espectral e índice de vegetacao em Paspalum Notatum Flügge vas. Notatum com visitas a modelagem de crescimento (Dissertacao de Mestrado). Porto Alegre: Pós-graduacao em Fitotecnia - Universidade Federal do Rio Grande do Sul.

Foster, A. J., Kakani, V. G., \& Mosali, J.

2016 "Estimation of bioenergy crop yield and N status by hyperspectral canopy reflectance and patrial least square regression". Precis. Agric., 1-18.

Georganos, S., Abdulhakim, A., Tenenbaum, D., \& Kalogirou, S. 2017 "Examining the NDVI - rainfall relationship in the semi-arid Sahel using geographically weigted regression". Journal of Arid Environments, 64-74.

Guilietti, A. M.

2003 "Diagnóstico da vegetacao nativa do bioma Caatinga". En J. M. SILVA, Biodiversidade da Caatinga: áreas e acoes prioritárias para a conservacao (págs. 51-73). Brasília: Ministério do Meio Ambiente.

Lemos, J. R.

2006 Floristica, estrutura e mapeamento da vegetacao de Caatinga da estacao ecológica de Ainaba - Ceará (tese de doutorado). Sao Paulo: Progrma de pós-graduacao em Ciencias Biologicas - Instituto de Biociencias - Universidade de Sao Paulo.

Lourenco, R. W., \& Landim, P. M.

2004 "Estudo da variabilidade do índice de vegetacao por diferenca normalizada - NDVI utilizando krigagem indicativa". Holos Environment, 38-55.

Major, M. S., Baret, F., \& Guyot, G. A. 1990 "Aratio vegetation index adjusted for soil brightness". Remote Sesing, 727-740.

Markahm, B. L., \& Barker, L. L.

1987 "Thematic mapper bandpass solar exoatmospherical irradiances". International Journal of Remote Senseing, 517-523. 
MMA.

2008 Estatistica florestal da Caatinga. Natal: Ministério do Meio Ambiente.

Myneni, R. B.

1995 "The mesaning os spectral vegetation indices". IEEE

Trans. Geosci. Remote Sensi., 481-486.

Nobrega, R. S., \& Santiago, G. C.

2014 "Tendencias de precipitacao na superficie do mar nos Oceanos Atlantico e Pacifico e Variabilidade de precipitacao em Pernambuco". Mercator, 107-118.

Oliveira, T. H.

2009 Balanco de radiacao da bacia hidrografica do rio Moxotó - PE através de imagens TM Landsat-5 e ETM Landsat-7 (Monografia). Recife : Departamento de Ciencias Geograficas - Universidade Federal de Pernambuco.

Oliveira, T., Galvíncio, J., Silva, J., Silva, C., Santiago, M. M., Menezes, J. B., \& Pimentel, R.

2009 Avaliação da Cobertura Vegetal e do Albedo da Bacia Hidrográfica do Rio Moxotó com Imagens do Satélite Landsat 5. XIV Simpósio Brasileiro de Sensoriamento Remoto, Anais, Natal: INPE, abril, 2865-2872.

Perazzo, G. M.

2002 Perfil do saneamento ambiental em 29 municipios da área de Xingó. Recife : Impresa Universitária.

Pernambuco, S.

2007 Diagnostico Florestal da regiao do Araripe. Recife: SECTMA.

Pinzon, J. E., \& Tucker, C. J.

2014 "A non-station 1981-2012 AVHRR NDVI3g time series. Remote Ses, 6929-6960.

Reis, A. A., Oliveira Sena , G. F., Walter, L. S., Lins, T. d., \&

Silva, E. A.

2017 Avaliacao Espaco-Temporal do desmatamento em paisagem do Sertao de Pernambuco. II CONIDIS II Congresso Internacional de Diversidade do Semiárido (págs. 1-12). Natal: CONDIS.

Riegelhaupt, E. M., \& Pareyn, F. G.

2010 "A questao energética e o manejo florestal da Caatinga". En M. A. Gariglio, Uso e conservacao dos recursos florestais da Caatinga (págs. 65-75). Brasília: Servico Florestal Brassileiro.

Rouse, J. W.

1974 Monitoring the vernal advancement and retogradation (green wave effect) of natural vegetation. Texas: Remote Sesing Center.

Silva, J. N. B. D., Albuquerque, J. V. D., \& Rodrigues, L. D. O.

2017 "Sustainable use of an extractivist reserve: a spatialtemporal analysis of environmental dynamics". Brazilian Journal of Biological Sciences, 4(7), 195-201.

Silva, H. A.

2009 Análise da cobertura utilizando ínidces de vegetacao e técnica de deteccao de mudancas na reserva biológica de Pedra Talhada PE/AL, con utilizacao de técnicas de sensoriamento remoto (Monografia). Refice : Departamento de Ciencias Geograficas - Unievrsidade Federal de Pernambuco.

Stöckeli, R., \& Vidale, P. L.

2004 "European plant phenology and climate as see in 20year AVHRR landsurface parameter dataset". International Journal of Remote Sensing, 3303-3330.

Stöckli, D. F.

2005 "Aplication of low-temperature thermochronometry to extensial tectonic setting". Mineralogy \& Geochemistry, 411-448.

Tian, F., Fensholt, R., Verbesselt, J., Grogan, K., Horion, S., \& Wang, $Y$.

2015 "Evaluating temporal cosistency of long-term global NDVI datasets for trend analysis". Remote Sens, 326-340.

Tucker, C. J.

1978 "A Comparison of satellite sensor bandes for vegetation monitoring". Photogranmetric Engineering and Remote Sensig, 1369-1380.

Tucker, C. J.

1991 "Mean and inter-year variation of growing season Normalized Difference Vegetation Index forthe Sahel 19811989”. International Journal of Remote Sensig, 1133-1137.

Zhao, Z., Goa, J., Wang, Y., Liu, J., \& Li, S.

2015 "Exploring spatially variable relationships between NDVI and climate factors in a trasition zone using geographically weighted regression". Theor. Appl. Climatol, 507-519.

\section{Nota}

1 Los "brejos" son humedales de altitud nordestinos que son enclaves de la Mata Atlántica, formando islas de forestas húmedas en plena región semiárida rodeados por vegetación de Caatinga, y tiene una condición climática bastante atípica con relación a la humedad, temperatura y vegetación y con poco conocimiento respecto de su vegetación y ecología (PORTO et al., 2004). 